\title{
Cyclostationary Analysis for Gearbox and Bearing Fault Diagnosis
}

\author{
Zhipeng Feng ${ }^{1}$ and Fulei $\mathrm{Chu}^{2}$ \\ ${ }^{1}$ School of Mechanical Engineering, University of Science and Technology Beijing, Beijing 100083, China \\ ${ }^{2}$ Department of Mechanical Engineering, Tsinghua University, Beijing 100084, China \\ Correspondence should be addressed to Zhipeng Feng; fengzp@ustb.edu.cn
}

Received 5 May 2015; Revised 24 July 2015; Accepted 27 July 2015

Academic Editor: Dong Wang

Copyright (c) 2015 Z. Feng and F. Chu. This is an open access article distributed under the Creative Commons Attribution License, which permits unrestricted use, distribution, and reproduction in any medium, provided the original work is properly cited.

\begin{abstract}
Gearbox and rolling element bearing vibration signals feature modulation, thus being cyclostationary. Therefore, the cyclic correlation and cyclic spectrum are suited to analyze their modulation characteristics and thereby extract gearbox and bearing fault symptoms. In order to thoroughly understand the cyclostationarity of gearbox and bearing vibrations, the explicit expressions of cyclic correlation and cyclic spectrum for amplitude modulation and frequency modulation (AM-FM) signals are derived, and their properties are summarized. The theoretical derivations are illustrated and validated by gearbox and bearing experimental signal analyses. The modulation characteristics caused by gearbox and bearing faults are extracted. In faulty gearbox and bearing cases, more peaks appear in cyclic correlation slice of 0 lag and cyclic spectrum, than in healthy cases. The gear and bearing faults are detected by checking the presence or monitoring the magnitude change of peaks in cyclic correlation and cyclic spectrum and are located according to the peak cyclic frequency locations or sideband frequency spacing.
\end{abstract}

\section{Introduction}

Gearboxes and rolling element bearings are critical mechanical components and widely used in many types of machinery [1-3]. Gear and bearing faults will result in deficiency of transmission or even shut-down of the entire machinery. Therefore, gearbox and bearing fault diagnosis play an important role.

The vibration signals of gearboxes and rolling element bearings are usually cyclostationary, since their statistics (in terms of ensemble average) change periodically with time due to their rotation. Hence, cyclostationary analysis is suitable to process gearbox and bearing vibration signals. Dalpiaz et al. [4] made a comparison study between various vibration signal analysis methods (including cepstrum, time-synchronous average, wavelet transform, and cyclostationary analysis) for gear localized fault detection, and they found spectral correlation density is effective in monitoring gear crack development. Sidahmed et al. $[5,6]$ showed that time-synchronous average can be considered as a first order cyclostationarity and spectral correlation as a second order cyclostationarity, found that gear vibration signals have second order cyclostationarity, and early detected gear tooth spalling using spectral correlation analysis. Zhu et al. [7] investigated the effectiveness of cyclostationarity from the first order to the third order, that is, spectrum of time-synchronous average, cyclic spectrum and cyclic bispectrum, in gearbox condition monitoring. Bi et al. [8] proposed to extract the amplitude modulation and phase modulation information from gear vibration signals using slice spectral correlation density, so as to detect gear defects. $\mathrm{Li}$ and $\mathrm{Qu}$ [9] deduced the cyclic correlation and cyclic spectrum of amplitude modulation signals and applied them to rolling element bearing fault diagnosis. Recently, Antoni et al. [10-15] conducted a series of researches on cyclostationary signal analysis and applied it to fault diagnosis of rotating machinery. To reduce the computational complexity of cyclic energy indicator based on cyclic spectral density, Wang and Shen [16] proposed 
an equivalent cyclic energy indicator for rolling element bearing degradation evaluation. These researches illustrate the effectiveness of cyclostationary analysis in gearbox and bearing fault diagnosis. However, the explicit relationship between the cyclostationary features of vibration signals and the gearbox and bearing dynamic nature still needs further investigation, in order for thoroughly understanding the vibration signal characteristics and thereby effectively diagnosing fault.

Gearbox and rolling element bearing vibration signals usually feature amplitude modulation and frequency modulation (AM-FM), and the modulation characteristics contain their health status information [17-21]. Cyclic correlation and cyclic spectrum are effective in extracting modulation features from amplitude modulation (AM), frequency modulation (FM), and AM-FM signals. Feng and his collaborators [22-24] derived the expressions of cyclic correlation and cyclic spectrum for gear AM-FM vibration signals and proposed indicators based on cyclic correlation and cyclic spectrum for detection and assessment of gearbox fault. Nevertheless, the carrier frequency of rolling element bearing vibration signals (resonance frequency) is completely different from that of gear vibration signals (gear meshing frequency and its harmonics). Therefore, it is important to investigate the cyclic correlation and cyclic spectrum of bearing vibration signals in depth, considering both the AM and the FM effects due to bearing fault. Meanwhile, how to explain the cyclostationary features displayed by the cyclic correlation and cyclic spectrum and to map the modulation characteristics to gear and bearing fault are still important issues for application of cyclostationary analysis in gearbox and bearing fault diagnosis. In this paper, we derive the explicit expressions of cyclic correlation and cyclic spectrum for general AM-FM signals, summarize their properties, and further extend the theoretical derivations to modulation analysis of both gear and rolling element bearing vibration signals, thus enabling cyclostationary analysis to detect and locate both gearbox and bearing fault.

\section{Cyclic Correlation}

2.1. Definition. The statistics of cyclostationary signals have periodicity or multiperiodicity with respect to time evolution. Cyclic statistics are suitable to process such signals. Among those, second order cyclic statistics, that is, cyclic correlation and cyclic spectrum, are effective in extracting the modulation features of cyclostationary signals.

For a signal $x(t)$, the cyclic autocorrelation function is defined as [25]

$$
R_{x}^{\alpha}(\tau)=\int_{-\infty}^{\infty} x\left(t+\frac{\tau}{2}\right)^{*} x\left(t-\frac{\tau}{2}\right) \exp (-j 2 \pi \alpha t) d t
$$

2.2. Cyclic Correlation of AM-FM Signal. During the constant speed running of gearboxes and rolling element bearings, the existence of fault, machining defect, and assembling error often leads to periodical changes in vibration signals. For gearboxes, such periodical changes modulate both the amplitude envelope and instantaneous frequency of gear meshing vibration. For bearings, such repeated changes excite resonance periodically. The excited resonance vanishes rapidly due to damping before next resonance comes, resulting in AM feature. Meanwhile, in one repeating cycle, the resonance exists in early period and the instantaneous frequency equals approximately the resonance frequency, while in later period, the resonance vanishes due to damping and the instantaneous frequency becomes 0 . That means the instantaneous frequency changes periodically, resulting in frequency modulation (FM). Hence the vibration signals of both gearboxes and rolling element bearings can be modeled as an AM-FM process $[17,21]$ plus a random noise

$$
x(t)=\sum_{k=0}^{K} a_{k}(t) \cos \left[2 \pi f_{c} t+b_{k}(t)+\theta_{k}\right]+\varepsilon(t),
$$

where

$$
\begin{aligned}
& a_{k}(t)=\sum_{n=0}^{N}\left(1+A_{k n}\right) \cos \left(2 \pi n f_{m} t+\phi_{k n}\right), \\
& b_{k}(t)=\sum_{l=0}^{L} B_{k i} \sin \left(2 \pi l f_{m} t+\varphi_{k l}\right)
\end{aligned}
$$

are the AM and FM functions, respectively, $A>0$ and $B>0$ are the magnitude of AM and FM respectively, $f_{c}$ is the carrier frequency (for gearboxes, it is the gear meshing frequency or its $k$ th harmonics; for rolling element bearings, it is the resonance frequency of bearing system), $f_{m}$ is the modulating frequency equal to the gear or bearing fault characteristic frequency, $\theta, \phi$, and $\varphi$ are the initial phase of AM and FM, respectively, and $\varepsilon(t)$ is a white Gaussian noise due to random background interferences.

Without loss of generality, consider only the fundamental frequency of the AM and FM terms; then (2) becomes

$$
\begin{aligned}
x(t)= & {\left[1+A \cos \left(2 \pi f_{m} t+\phi\right)\right] } \\
& \cdot \cos \left[2 \pi f_{c} t+B \sin \left(2 \pi f_{m} t+\varphi\right)+\theta\right]+\varepsilon(t) .
\end{aligned}
$$

According to the identity [26]

$$
\exp [j z \sin (\beta)]=\sum_{k=-\infty}^{\infty} J_{k}(z) \exp (j k \beta),
$$

where $J_{k}(z)$ is Bessel function of the first kind with integer order $k$ and argument $z$, the FM term in (5) can be expanded as a Bessel series, and then (5) becomes

$$
\begin{aligned}
x(t)= & {\left[1+A \cos \left(2 \pi f_{m} t+\phi\right)\right] } \\
& \cdot \sum_{n=-\infty}^{\infty} J_{n}(B) \cos \left(2 \pi f_{c} t+2 \pi n f_{m} t+n \varphi+\theta\right) \\
& +\varepsilon(t) .
\end{aligned}
$$


For such a signal expressed as (7), the time-varying feature of its autocorrelation function is mainly determined by the AM-FM part, since the autocorrelation function of a white Gaussian noise $R_{\varepsilon}(\tau)=\delta(\tau)$ (i.e., its Fourier transform has peak at 0 only, and therefore the noise $\varepsilon(t)$ does not affect identification of modulating frequency via cyclic correlation and cyclic spectrum analysis). In addition, it is the AM and
FM effects on the carrier signal that leads to the cyclostationarity of bearing and gearbox vibration signals, and we rely on detection of the modulating frequency of such AM and FM effects to diagnose bearing and gearbox fault. Therefore, we neglect the noise $\varepsilon(t)$ and focus on the deterministic AM-FM part only in the following analysis. Then the cyclic autocorrelation function of (7) can be derived as [22]

$$
R_{x}^{\alpha}(\tau)= \begin{cases}\frac{1}{2}\left[h_{L}(0,0)+\frac{1}{4} A^{2} h_{L}\left(-2 f_{m}, 0\right)+\frac{1}{4} A^{2} h_{L}\left(2 f_{m}, 0\right)\right], & \alpha= \pm\left(n-n^{\prime}\right) f_{m} \\ \frac{1}{4} A\left[h_{L}\left(\mp f_{m}, \pm \phi\right)+h_{L}\left( \pm f_{m}, \pm \phi\right)\right], & \alpha= \pm\left(n-n^{\prime} \pm 1\right) f_{m} \\ \frac{1}{8} A^{2} h_{L}(0, \pm 2 \phi), & \alpha= \pm\left(n-n^{\prime} \pm 2\right) f_{m}\end{cases}
$$

in lower cyclic frequency domain, and

$$
R_{x}^{\alpha}(\tau)= \begin{cases}\frac{1}{2}\left[h_{H}(0,0)+\frac{1}{4} A^{2} h_{H}\left(-2 f_{m}, 0\right)+\frac{1}{4} A^{2} h_{H}\left(2 f_{m}, 0\right)\right], & \alpha= \pm\left[2 f_{c}+\left(n+n^{\prime}\right) f_{m}\right], \\ \frac{1}{4} A\left[h_{H}\left(\mp f_{m}, \pm \phi\right)+h_{H}\left( \pm f_{m}, \pm \phi\right)\right], & \alpha= \pm\left[2 f_{c}+\left(n+n^{\prime} \pm 1\right) f_{m}\right], \\ \frac{1}{8} A^{2} h_{H}(0, \pm 2 \phi), & \alpha= \pm\left[2 f_{c}+\left(n+n^{\prime} \pm 2\right) f_{m}\right]\end{cases}
$$

in higher cyclic frequency domain, where the intermediate functions

$$
\begin{aligned}
& h_{L}(\eta, \psi)=\sum_{n=-\infty}^{\infty} \sum_{n^{\prime}=-\infty}^{\infty} J_{n}(B) J_{n^{\prime}}(B) \\
& \cdot \exp (\left. \pm j\left\{\pi\left[2 f_{c}+\left(n+n^{\prime}\right) f_{m}+\eta\right] \tau+\left(n-n^{\prime}\right) \varphi+\psi\right\}\right), \\
& h_{H}(\eta, \psi)=\sum_{n=-\infty}^{\infty} \sum_{n^{\prime}=-\infty}^{\infty} J_{n}(B) J_{n^{\prime}}(B) \\
& \cdot \exp \left( \pm j\left\{\pi\left[\left(n-n^{\prime}\right) f_{m}+\eta\right] \tau+\left(n+n^{\prime}\right) \varphi+2 \theta+\psi\right\}\right) .
\end{aligned}
$$

Set the time lag to 0 , yielding the slice of cyclic autocorrelation function

$$
R_{x}^{\alpha}(0)= \begin{cases}\left(\frac{1}{2}+\frac{1}{4} A^{2}\right) h_{L 0}(0), & \alpha= \pm\left(n-n^{\prime}\right) f_{m}, \\ \frac{1}{2} A h_{L 0}( \pm \phi), & \alpha= \pm\left(n-n^{\prime} \pm 1\right) f_{m}, \\ \frac{1}{8} A^{2} h_{L 0}( \pm 2 \phi), & \alpha= \pm\left(n-n^{\prime} \pm 2\right) f_{m},\end{cases}
$$

$$
\begin{aligned}
& R_{x}^{\alpha}(0) \\
& = \begin{cases}\frac{1}{2}\left(1+\frac{1}{2} A^{2}\right) h_{H 0}(0), & \alpha= \pm\left[2 f_{c}+\left(n+n^{\prime}\right) f_{m}\right], \\
\frac{1}{2} A h_{H 0}( \pm \phi), & \alpha= \pm\left[2 f_{c}+\left(n+n^{\prime} \pm 1\right) f_{m}\right], \\
\frac{1}{8} A^{2} h_{H 0}( \pm 2 \phi), & \alpha= \pm\left[2 f_{c}+\left(n+n^{\prime} \pm 2\right) f_{m}\right],\end{cases}
\end{aligned}
$$

in lower and higher cyclic frequency domains, respectively, where the intermediate functions

$$
\begin{gathered}
h_{L 0}(\psi)=\sum_{n=-\infty}^{\infty} \sum_{n^{\prime}=-\infty}^{\infty} J_{n}(B) J_{n^{\prime}}(B) \\
\cdot \exp \left\{ \pm j\left[\left(n-n^{\prime}\right) \varphi+\psi\right]\right\}, \\
h_{H 0}(\psi)=\sum_{n=-\infty}^{\infty} \sum_{n^{\prime}=-\infty}^{\infty} J_{n}(B) J_{n^{\prime}}(B) \\
\cdot \exp \left\{ \pm j\left[\left(n+n^{\prime}\right) \varphi+2 \theta+\psi\right]\right\} .
\end{gathered}
$$

According to (10a) and (10b) and (11a) and (11b), the slice of cyclic autocorrelation function has two clusters of cyclic frequencies: one cluster concentrates around the cyclic frequency of $0 \mathrm{~Hz}$ separated by the modulating frequency $f_{m}$, and the other cluster spreads around twice the carrier 
frequency $2 f_{c}$ with a spacing equal to the modulating frequency $f_{m}$.

In lower cyclic frequency domain, the cyclic frequency locations of present peaks are dependent on the difference of the two Bessel function orders $n-n^{\prime}$. For any peak at a specific cyclic frequency, $n-n^{\prime}=$ constant. This leads to constant complex exponentials in (11a). Furthermore, according to the addition theorem of Bessel functions [26]

$$
\sum_{n=-\infty}^{\infty} \sum_{n^{\prime}=-\infty}^{\infty} J_{n}(z) J_{n^{\prime}}(z)= \begin{cases}1, & n=n^{\prime}, \\ 0, & n \neq n^{\prime}\end{cases}
$$

peaks appear in the slice of cyclic autocorrelation function in lower cyclic frequency domain, if and only if the orders of the two Bessel functions are equal to each other; that is, $n=n^{\prime}$. Therefore, the cyclic autocorrelation slice in lower cyclic frequency domain can be further simplified as

$$
R_{x}^{\alpha}(0)= \begin{cases}\frac{1}{2}+\frac{1}{4} A^{2}, & \alpha=0, \\ \frac{1}{2} A \exp ( \pm j \phi), & \alpha= \pm f_{m} \\ \frac{1}{8} A^{2} \exp ( \pm j 2 \phi), & \alpha= \pm 2 f_{m} .\end{cases}
$$

According to (13), in lower cyclic frequency domain of the cyclic autocorrelation slice, peaks appear at the cyclic frequencies of $0 \mathrm{~Hz}$, the modulating frequency $f_{m}$, and its twice $2 f_{m}$. If higher order harmonics of the modulating frequency are taken into account, then peaks also appear at the modulating frequency harmonics $k f_{m}$.

The addition theorem of Bessel functions does not apply to the slice of cyclic autocorrelation function in higher cyclic frequency domain. The cyclic frequency locations of present peaks are dependent on the sum of the two Bessel function orders $n+n^{\prime}$. For any specific peak, $n+n^{\prime}=$ constant. This does not mean $n-n^{\prime}=$ constant. According to (10b) and (11b), in higher cyclic frequency domain of the cyclic autocorrelation slice, sidebands appear at both sides of twice the carrier frequency $2 f_{c}$, with a spacing equal to the modulating frequency $f_{m}$.

According to the above derivations, we can detect gearbox and bearing fault by monitoring the presence or magnitude change of sidebands around the cyclic frequency of 0 or twice the carrier frequency, that is, twice the meshing frequency for gearboxes and twice the resonance frequency for bearings, in the 0 lag slice of cyclic autocorrelation function. We can further locate the gearbox and bearing fault by matching the sideband spacing with the fault characteristic frequencies.

\section{Cyclic Spectrum}

3.1. Definition. For a signal $x(t)$, the cyclic spectral density is defined as the Fourier transform of the cyclic correlation function [25]

$$
S_{x}^{\alpha}(f)=\int_{-\infty}^{\infty} R_{x}^{\alpha}(\tau) \exp (-j 2 \pi f \tau) d \tau
$$

where $f$ is frequency.

3.2. Cyclic Spectrum of AM-FM Signal. Without loss of generality, we still consider the simplified gearbox and bearing vibration signal model, (5), by focusing on the fundamental frequency of the AM and FM terms. Its cyclic spectral density can be derived as $[23,24]$

$$
\begin{aligned}
& S_{x}^{\alpha}(f)= \begin{cases}\frac{1}{2} H(0), & \alpha=\left(n-n^{\prime}\right) f_{m}, f=f_{c}+\frac{1}{2}\left(n+n^{\prime}\right) f_{m}, \\
\frac{1}{8} A^{2} H(0), & \alpha=\left(n-n^{\prime}\right) f_{m}, f=f_{c}+\frac{1}{2}\left(n+n^{\prime} \mp 2\right) f_{m}, \\
\frac{1}{4} A H( \pm \phi), & \alpha=\left(n-n^{\prime} \pm 1\right) f_{m}, f=f_{c}+\frac{1}{2}\left(n+n^{\prime} \mp 1\right) f_{m}, \\
\frac{1}{8} A^{2} H(2 \phi), & \alpha=\left(n-n^{\prime} \pm 2\right) f_{m}, f=f_{c}+\frac{1}{2}\left(n+n^{\prime}\right) f_{m},\end{cases} \\
& S_{x}^{\alpha}(f)=\left\{\begin{array}{lc}
\frac{1}{2} H(2 \theta), & \alpha=2 f_{c}+\left(n+n^{\prime}\right) f_{m}, f=\frac{1}{2}\left(n-n^{\prime}\right) f_{m}, \\
\frac{1}{8} A^{2} H(2 \theta), & \alpha=2 f_{c}+\left(n+n^{\prime}\right) f_{m}, f=\frac{1}{2}\left(n-n^{\prime} \mp 2\right) f_{m}, \\
\frac{1}{4} A H(2 \theta \pm \phi), & \alpha=2 f_{c}+\left(n+n^{\prime} \pm 1\right) f_{m}, f=\frac{1}{2}\left(n-n^{\prime} \mp 1\right) f_{m}, \\
\frac{1}{8} A^{2} H(2 \theta \pm 2 \phi), & \alpha=2 f_{c}+\left(n+n^{\prime} \pm 2\right) f_{m}, f=\frac{1}{2}\left(n-n^{\prime}\right) f_{m},
\end{array}\right.
\end{aligned}
$$


in lower and higher cyclic frequency domains, respectively, where the intermediate function

$$
H(\psi)=\sum_{n=-\infty}^{\infty} \sum_{n^{\prime}=-\infty}^{\infty} J_{n}(B) J_{n^{\prime}}(B) \exp ( \pm j \psi) .
$$

According to (15a) and (15b), peaks appear at specific locations only on the cyclic frequency-frequency plane. Their cyclic frequency locations in lower cyclic frequency domain, as well as their frequency locations in higher cyclic frequency domain, are dependent on the difference of the two Bessel function orders $n-n^{\prime}$. For any specific peaks, $n-n^{\prime}=$ constant. Then, the addition theorem of Bessel functions [26], (12), applies to the cyclic spectral density, (15a) and (15b). Hence, on the cyclic frequency-frequency plane of the cyclic spectral density, peaks appear if and only if the orders of the two Bessel functions are equal to each other; that is, $n=n^{\prime}$. Then cyclic spectral density can be simplified as

$$
\begin{aligned}
& S_{x}^{\alpha}(f)=J_{n}^{2}(B) \\
& \qquad \begin{cases}\frac{1}{2}, & \alpha=0, f=f_{c}+n f_{m}, \\
\frac{1}{8} A^{2}, & \alpha=0, f=f_{c}+(n \mp 1) f_{m}, \\
\frac{1}{4} A \exp ( \pm j \phi), & \alpha= \pm f_{m}, f=f_{c}+\left(n \mp \frac{1}{2}\right) f_{m}, \\
\frac{1}{8} A^{2} \exp ( \pm j 2 \phi), & \alpha= \pm 2 f_{m}, f=f_{c}+n f_{m},\end{cases}
\end{aligned}
$$

in lower cyclic frequency domain and

$$
S_{x}^{\alpha}(f)=J_{n}^{2}(B) \begin{cases}\frac{1}{2} \exp ( \pm j 2 \theta), & \alpha=2 f_{c}+2 n f_{m}, f=0, \\ \frac{1}{8} A^{2} \exp ( \pm j 2 \theta), & \alpha=2 f_{c}+2 n f_{m}, f=\mp f_{m}, \\ \frac{1}{4} A \exp [ \pm j(2 \theta \pm \phi)], & \alpha=2 f_{c}+(2 n \pm 1) f_{m}, f=\mp \frac{1}{2} f_{m}, \\ \frac{1}{8} A^{2} \exp [ \pm j(2 \theta \pm 2 \phi)], & \alpha=2 f_{c}+(2 n \pm 2) f_{m}, f=0,\end{cases}
$$

in higher cyclic frequency domain.

According to (17a) and (17b), for any peak on the cyclic frequency-frequency plane, its frequency location in lower cyclic frequency domain, as well as its cyclic frequency location in higher cyclic frequency domain, is dependent on the Bessel function order $n$. Meanwhile, the peak magnitude only involves a few Bessel functions. Thus (17a) and (17b) can be further simplified as

$$
\begin{aligned}
& S_{x}^{\alpha}(f)= \begin{cases}\frac{1}{2} J_{n}^{2}(B)+\frac{1}{8} A^{2}\left[J_{n-1}^{2}(B)+J_{n+1}^{2}(B)\right], & \alpha=0, f=f_{c}+n f_{m}, \\
\frac{1}{4} A\left[J_{n}^{2}(B)+J_{n+1}^{2}(B)\right] \exp ( \pm j \phi), & \alpha= \pm f_{m}, f=f_{c}+\left(n+\frac{1}{2}\right) f_{m}, \\
\frac{1}{8} A^{2} J_{n}^{2}(B) \exp ( \pm j 2 \phi), & \alpha= \pm 2 f_{m}, f=f_{c}+n f_{m},\end{cases} \\
& S_{x}^{\alpha}(f)= \begin{cases}\frac{1}{2} J_{n}^{2}(B)+\frac{1}{8} A^{2}\left[J_{n-1}^{2}(B)+J_{n+1}^{2}(B)\right] \exp ( \pm j 2 \theta), & \alpha=2 f_{c}+2 n f_{m}, f=0, \\
\frac{1}{4} A\left[J_{n}^{2}(B)+J_{n+1}^{2}(B)\right] \exp [ \pm j(2 \theta \pm \phi)], & \alpha=2 f_{c}+(2 n+1) f_{m}, f=\mp \frac{1}{2} f_{m}, \\
\frac{1}{8} A^{2} J_{n}^{2}(B) \exp [ \pm j(2 \theta \pm 2 \phi)], & \alpha=2 f_{c}+2 n f_{m}, f=\mp f_{m},\end{cases}
\end{aligned}
$$

in lower and higher cyclic frequency domains, respectively.

According to (18a), in lower cyclic frequency domain, peaks appear at the cyclic frequencies of $0 \mathrm{~Hz}$, modulating frequency $f_{m}$, and its twice $2 f_{m}$. If higher order harmonics of modulating frequency are taken into account, then peaks also appear at the cyclic frequencies of the modulating frequency harmonics $k f_{m}$. Along the frequency axis, these peaks center around the carrier frequency $f_{c}$, with a spacing equal to the modulating frequency $f_{m}$.

According to (18b), in higher cyclic frequency domain, peaks appear at the frequencies of $0 \mathrm{~Hz}$, modulating frequency $f_{m}$, and its half $1 / 2 f_{m}$. If higher order harmonics of modulating frequency are taken into account, then peaks also appear at the modulating frequency harmonics $k f_{m}$ and 


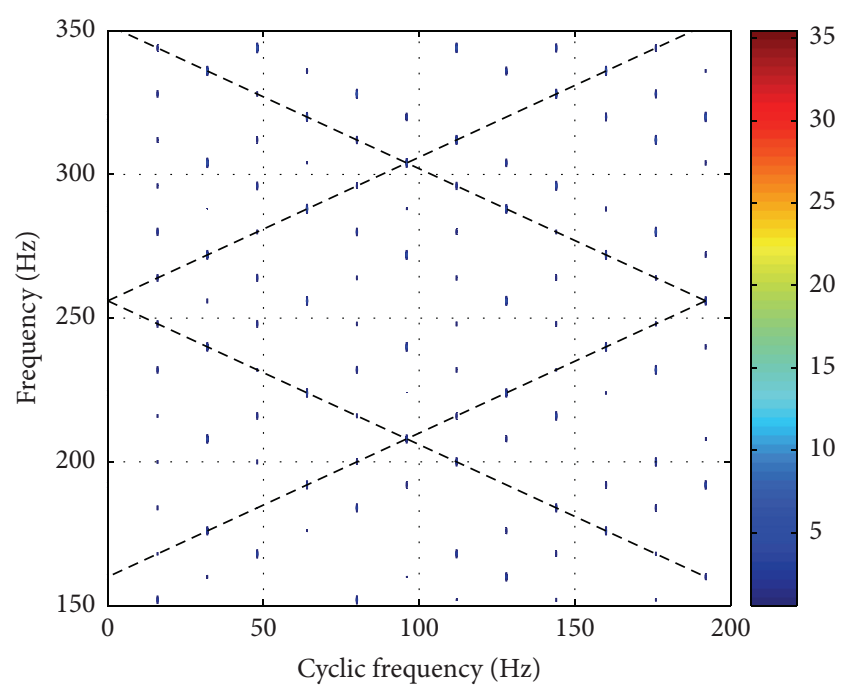

FIGURE 1: Cyclic spectrum distribution characteristics of an AM-FM signal.

their half $k / 2 f_{m}$. Along the cyclic frequency axis, these peaks center around twice the carrier frequency $2 f_{c}$, with a spacing equal to twice the modulating frequency $2 f_{m}$.

Observing the peak locations in (18a) and (18b), the peaks in cyclic spectrum distribute along the lines

$$
f= \pm \frac{\alpha}{2} \pm\left(f_{c} \pm n f_{m}\right) .
$$

These lines form diamonds on the cyclic frequency-frequency plane, as illustrated by Figure 1, which is the cyclic spectrum of an AM-FM signal with a carrier frequency of $256 \mathrm{~Hz}$ and a modulating frequency of $16 \mathrm{~Hz}$.

According to the above derivations, we can detect gearbox and bearing fault by monitoring the presence or magnitude change of peaks in the cyclic spectrum. For example, in lower cyclic frequency band, focus on the points corresponding to the cyclic frequency locations of modulating frequency $f_{m}$ and its multiples $k f_{m}$ and to the frequency locations of the carrier frequency plus the modulating frequency multiples $f_{c}+n f_{m}$. We can further locate the gearbox and bearing fault by matching the cyclic frequency spacing of peaks with the fault characteristic frequencies.

\section{Gearbox Signal Analysis}

4.1. Specification of Experiment. Figure 2 shows the experimental system. The gearbox has one gear pair. Table 1 lists the configuration and running condition of the gearbox. Two statuses of the gearbox are simulated. Under the healthy status, the gear pair is perfect. While under the faulty status, one of the pinion teeth is spalled, whereas the gear is perfect. During the experiment, accelerometer signals are collected at a sampling frequency of $20000 \mathrm{~Hz}$ and each record lasts for $3 \mathrm{~s}$ (i.e., 60000 samples) which covers more than 51 and 48 revolutions for the drive pinion and the driven

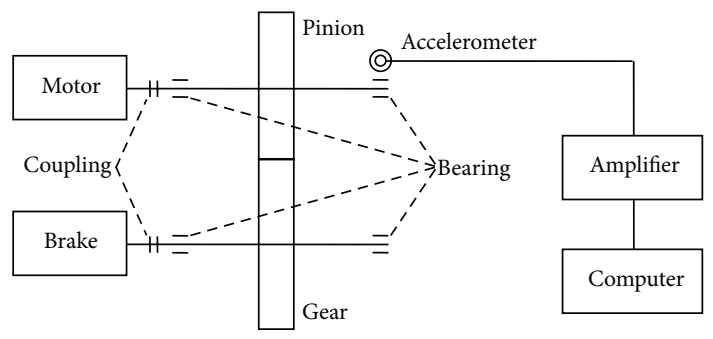

FIGURE 2: Gearbox experimental setup.

TABLE 1: Gearbox configuration and running condition.

\begin{tabular}{|c|c|c|}
\hline & Drive pinion & Driven gear \\
\hline Number of teeth & 20 & 21 \\
\hline Rotating frequency $(\mathrm{Hz})$ & 17.285 & 16.462 \\
\hline Meshing frequency $(\mathrm{Hz})$ & \multicolumn{2}{|c|}{345.7} \\
\hline Brake torque $(\mathrm{Nm})$ & - & 200 \\
\hline
\end{tabular}

gear, respectively, and is long enough to reveal the cyclostationarity.

4.2. Signal Analysis. Figure 3 shows the waveform, Fourier spectrum, cyclic correlation 0 lag slice, and cyclic spectrum (in lower cyclic frequency band) of the healthy gearbox. In the following analysis, the peak frequencies do not correspond exactly to those in Table 1 . This is reasonable, because the actual speed is inevitably different from the set one in real experiments. In the cyclic correlation slice, Figure 3(c), most of the present peaks correspond to the driven gear rotating frequency harmonics. Although a few peaks appear at the pinion and gear characteristic frequencies and their harmonics, their magnitudes are not strong. In the cyclic spectrum (the colorbar on the right shows the magnitude, the same setting in the following cyclic spectra), Figure 3(d), along the frequency axis, the present peaks center around the gear meshing frequency $345.7 \mathrm{~Hz}$, and its harmonics, and along the cyclic axis, they appear at the pinion and gear characteristic frequencies and their harmonics. The presence of these peaks is reasonable; since gear manufacturing errors and minor defects are inevitable, they will result in these peaks.

Figure 4 shows the waveform, Fourier spectrum, cyclic correlation 0 lag slice, and cyclic spectrum (in lower cyclic frequency band) of the faulty gearbox. In the cyclic correlation slice, Figure 4(c), peaks appear at the pinion and gear characteristic frequencies and their harmonics. Except the first two peaks, nearly all the other peaks appear at the cyclic frequency locations of the drive pinion rotating frequency harmonics, and they are higher than those in that of the healthy gearbox. In the cyclic spectrum, Figure 4(d), more and stronger peaks appear than in that of the healthy gearbox. Figure 4(e) shows the zoomed-in cyclic spectrum, for revealing the peak distribution details. Along the cyclic frequency axis, peaks appear along lines corresponding to the pinion 


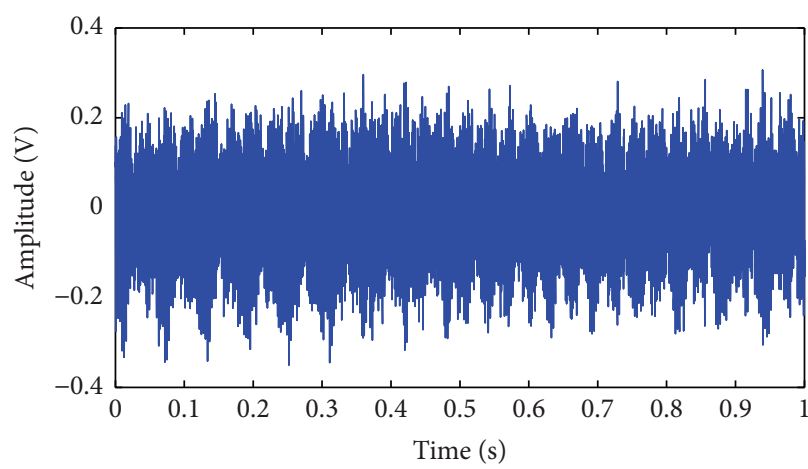

(a) Waveform

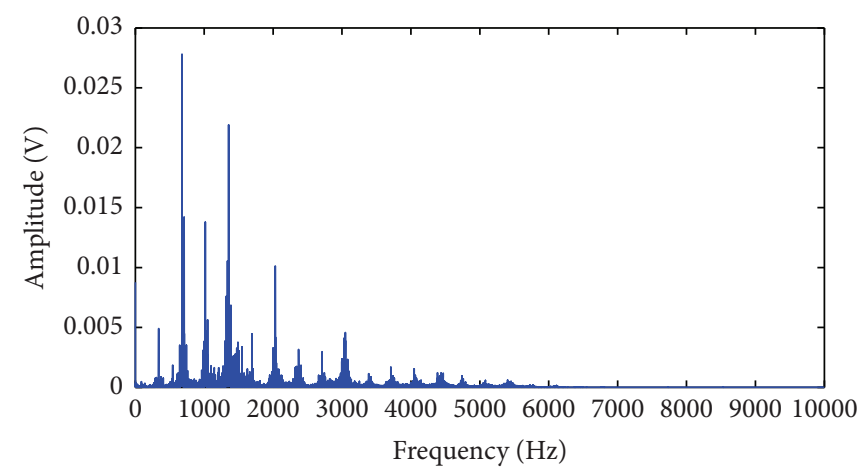

(b) Fourier spectrum

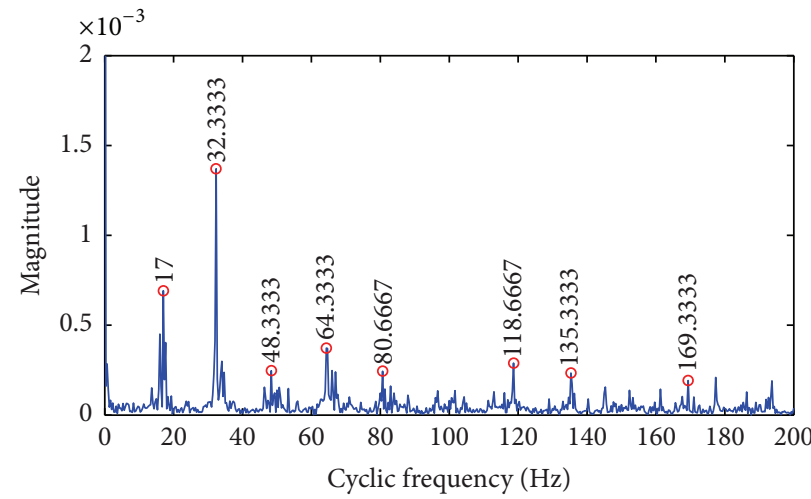

(c) Cyclic correlation 0 lag slice

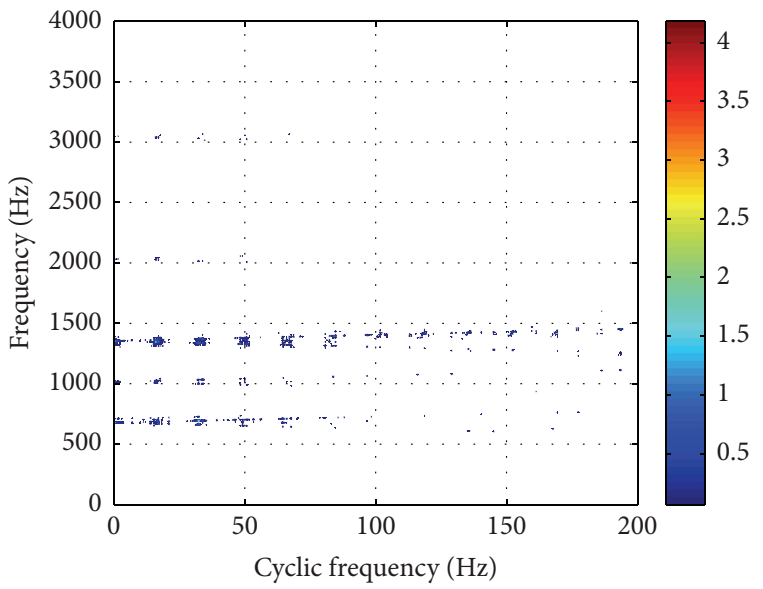

(d) Cyclic spectrum

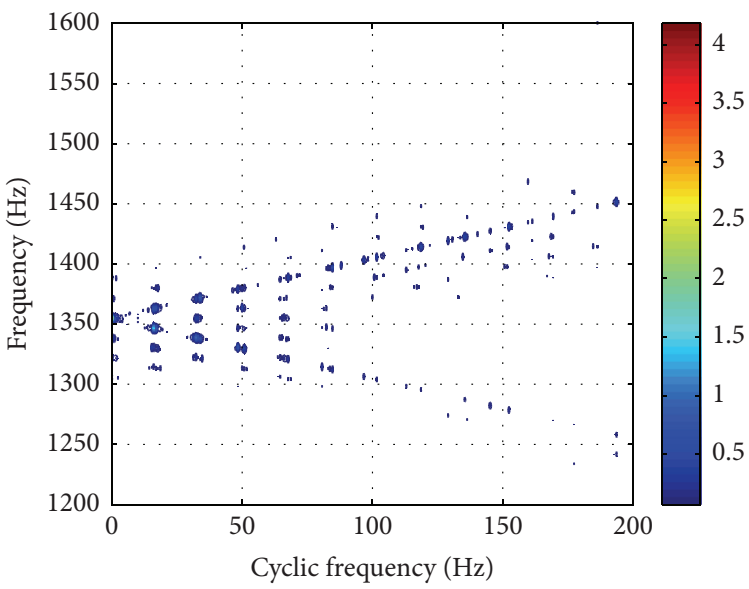

(e) Zoomed-in cyclic spectrum

Figure 3: Healthy gearbox signal.

characteristic frequency and its multiples. While along the frequency axis, sidebands appear around $1400 \mathrm{~Hz}$ about four times the gear meshing frequency, with a spacing equal to the pinion characteristic frequency. Based on the theoretical derivations in Sections 2 and 3, the cyclic frequency locations of peaks in cyclic correlation slice and cyclic spectrum, as well as the sideband spacing along frequency axis in cyclic spectrum, relate to the modulating frequency of AM-FM signals. These features imply that more harmonics of the drive pinion rotating frequency get involved in modulating the gear meshing vibration and that the drive pinion has a stronger modulation effect than the driven gear, indicating the pinion fault. These findings are consistent with the actual condition of the faulty gearbox.

\section{Bearing Signal Analysis}

5.1. Specification of Experiment. Figure 5 shows the experimental setup of the test [27]. A shaft is supported by four 


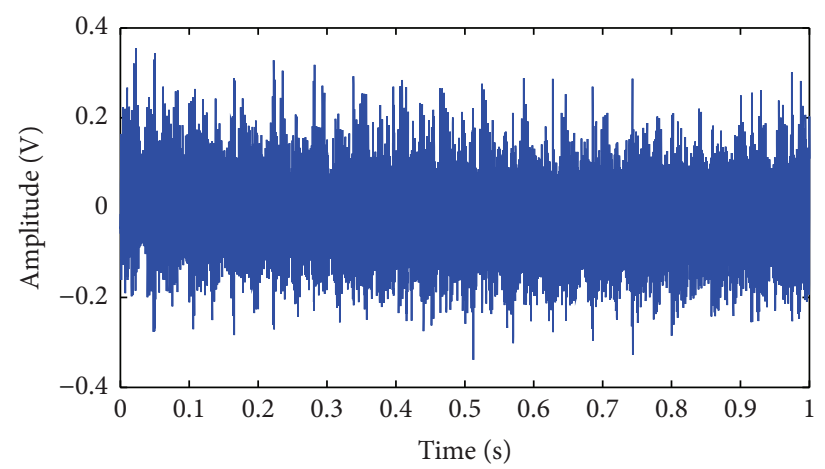

(a) Waveform

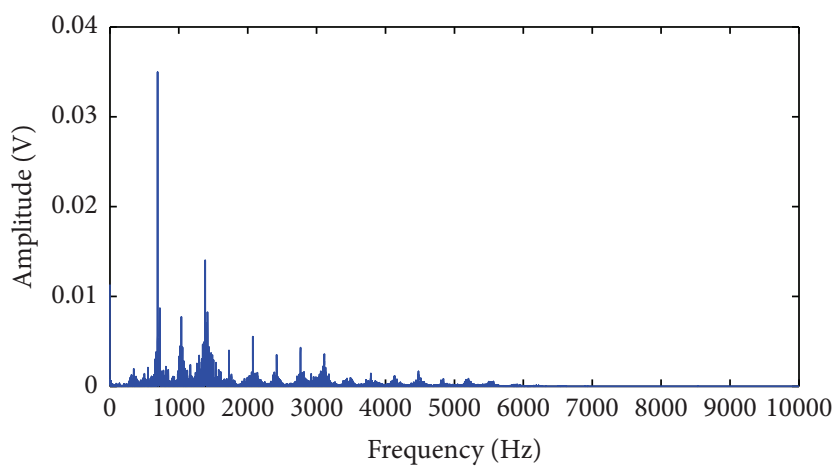

(b) Fourier spectrum

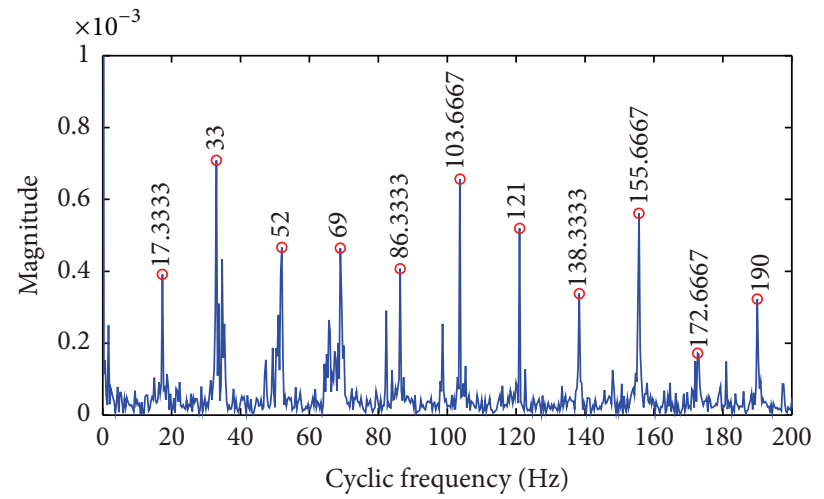

(c) Cyclic correlation 0 lag slice

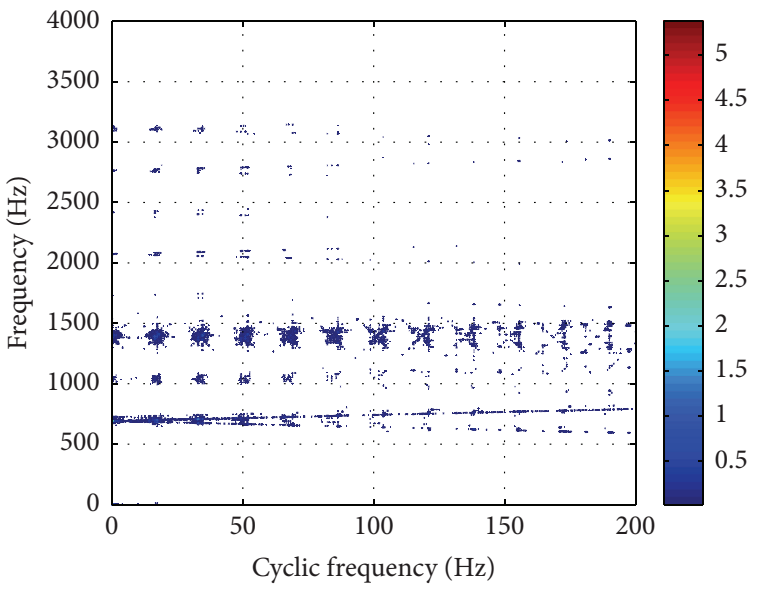

(d) Cyclic spectrum

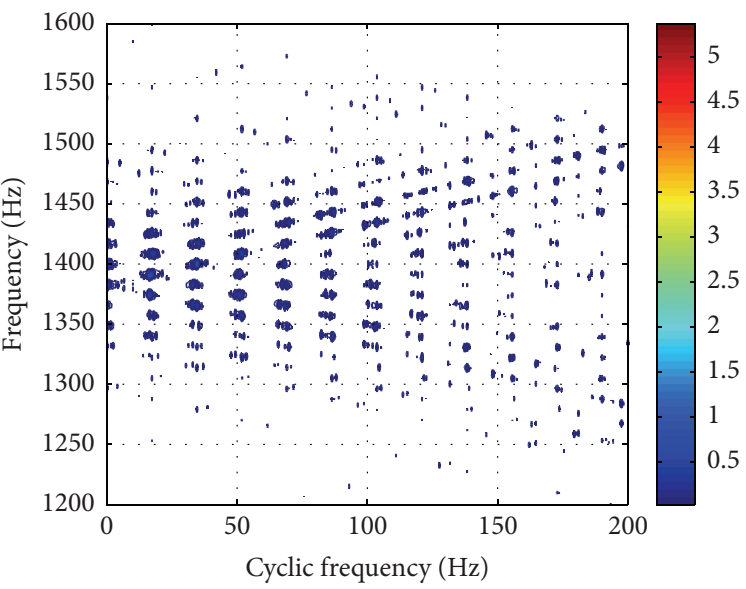

(e) Zoomed-in cyclic spectrum

FIGURE 4: Faulty gearbox signal.

Rexnord ZA-2115 double row rolling element bearings and is driven by an AC motor through rub belts at a speed of $2000 \mathrm{rpm}$. A radial load of $6000 \mathrm{lbs}$ is applied to the shaft and bearings by a spring mechanism. The bearing test was kept running for eight days until damage occurs to the outer race of bearing 1 . In the normal case, all the four bearings are healthy. While in the faulty case, the outer race of bearing 1 has damage, as shown in Figure 6. Accelerometers are mounted on the bearing housings, and the vibration signals are collected at a sampling frequency of $20480 \mathrm{~Hz}$, and 20480 data points are recorded for both the healthy and faulty cases. The main parameters of the four bearings are listed in Table 2. The characteristic frequency of each element fault is calculated and listed in Table 3.

5.2. Signal Analysis. Figure 7 shows the waveform, Fourier spectrum, cyclic correlation 0 lag slice, and cyclic spectrum 


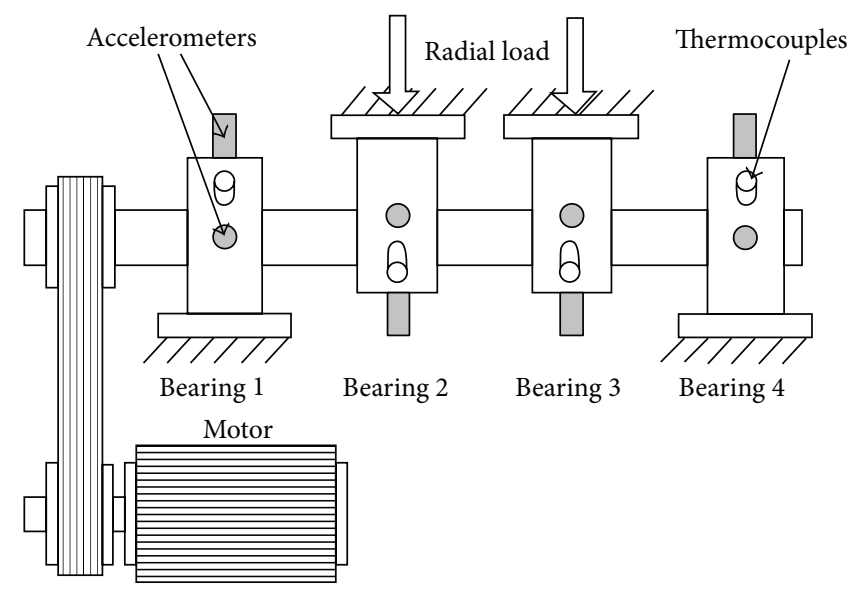

FIgURE 5: Bearing experimental setup [27].

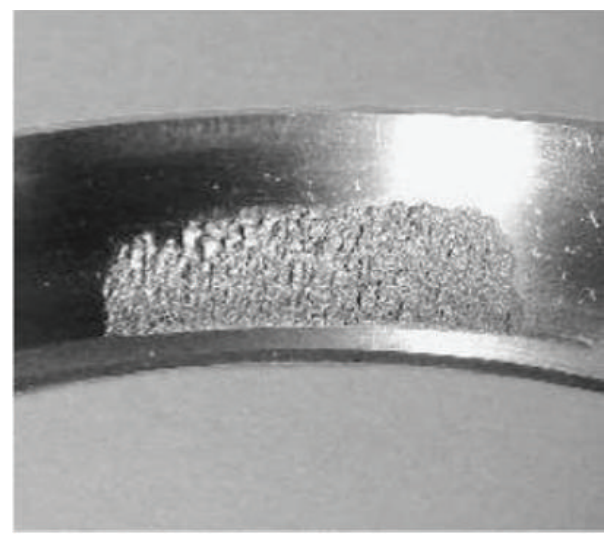

FIgURE 6: Outer race damage of bearing 1 [27].

TABle 2: Parameters of bearing Rexnord ZA-2115.

\begin{tabular}{lccc}
\hline $\begin{array}{l}\text { Number of } \\
\text { rollers }\end{array}$ & Roller diameter & Pitch diameter & Contact angle \\
\hline 16 & $0.331[\mathrm{in}]$ & $2.815[\mathrm{in}]$ & $15.17\left[^{\circ}\right]$ \\
\hline
\end{tabular}

TABLE 3: Characteristic frequency of bearing Rexnord ZA-2115 [Hz].

\begin{tabular}{lccr}
\hline Outer race & Inner race & \multicolumn{2}{c}{ Roller } \\
\hline 236.404 & 296.930 & 139.917 & 279.833 \\
\hline
\end{tabular}

(in lower cyclic frequency band) of the normal case. In the cyclic correlation slice, Figure 7(c), a few small peaks appear, but they do not correspond to any bearing component fault characteristic frequency or its multiples. Although some peaks appear in the cyclic spectrum, Figure $7(\mathrm{~d})$, they do not distribute along lines corresponding to the cyclic frequencies of any bearing component fault characteristic frequency or its multiples. These features imply that no significant modulation on the resonance vibration and the bearing is healthy.
Figure 8 shows the waveform, Fourier spectrum, cyclic correlation 0 lag slice, and cyclic spectrum (in lower cyclic frequency band) of the faulty case. In the close-up view of the Fourier spectrum, some sidebands appear, but the frequency spacing is not identically equal to the outer race fault characteristic frequency. In the cyclic correlation slice, Figure 8(d), more peaks appear and their magnitudes are higher than in the normal case. Moreover, their cyclic frequency locations correspond to the outer race fault characteristic frequency and multiples up to the 25th order. In the cyclic spectrum, Figure 8(e), many peaks are present. More importantly, they distribute along lines associated with the outer race fault characteristic frequency and multiples. At any cyclic frequency of the outer race fault characteristic frequency or its multiple, these peaks form sidebands along the frequency axis, with a spacing equal to the outer race fault characteristic frequency. For example, in the zoomed-in cyclic spectrum, Figure 8(f), the four peaks labeled by capitals A, B, C, and D distribute along a line at the cyclic frequency of $710 \mathrm{~Hz}$ (about three times the outer race fault characteristic frequency), and they have a spacing of $236 \mathrm{~Hz}$ (approximately equal to the outer race fault characteristic frequency) along the frequency axis. These findings imply that the outer race characteristics 


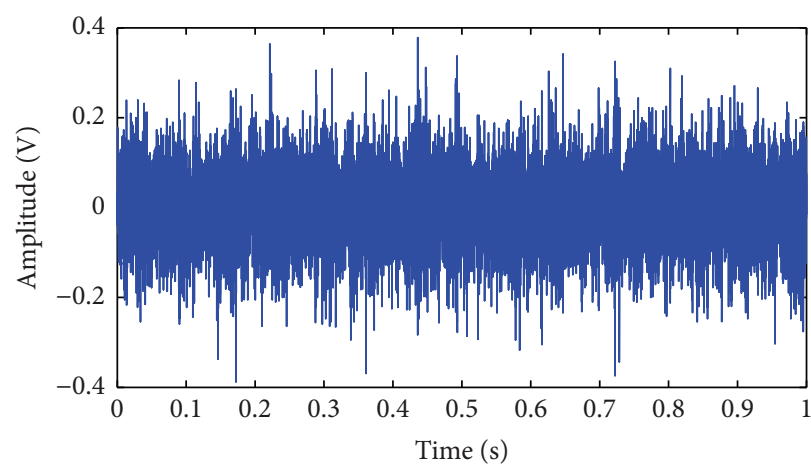

(a) Waveform

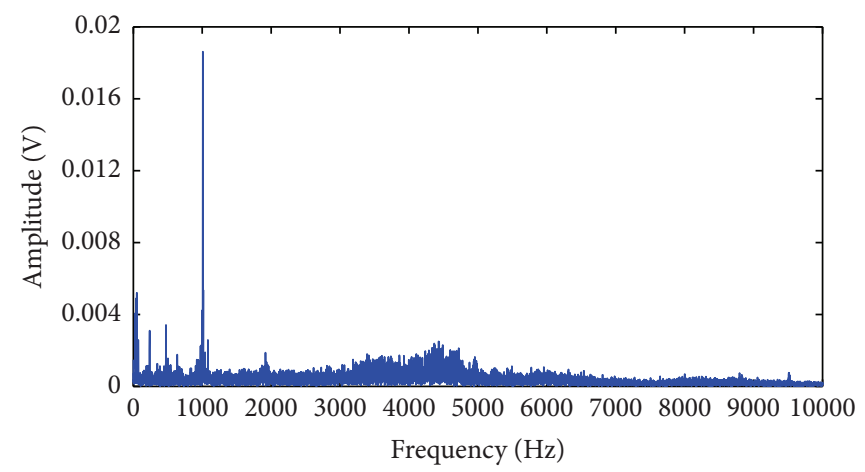

(b) Fourier spectrum

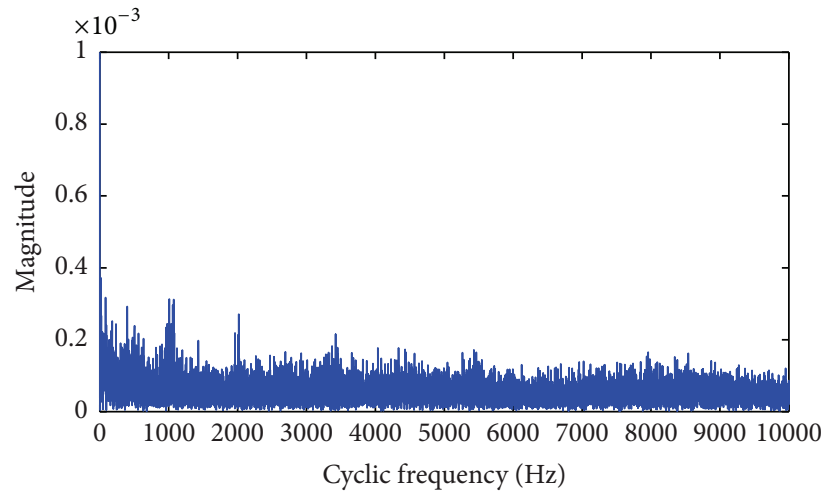

(c) Cyclic correlation 0 lag slice

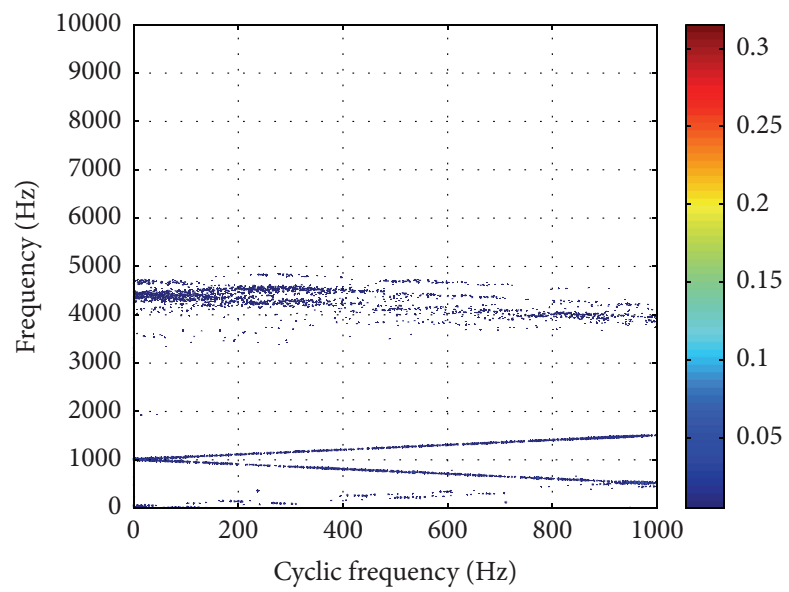

(d) Cyclic spectrum

FIGURE 7: Normal bearing signal.

frequency has strong modulation effect on the resonance, indicating fault existence on the bearing outer race, in accordance with the actual settings.

\section{Discussion and Conclusions}

Since gearbox and rolling element bearing vibration signals are characterized by AM-FM feature, their Fourier spectra have complex sideband structure due to the convolution between the Fourier spectra of AM and FM parts as well as the infinite Bessel series expansion of an FM term. Although gearbox and bearing fault can be identified via conventional Fourier spectrum, it involves complex sideband analysis and relies on the sideband spacing to extract the fault characteristic frequency.

Considering the cyclostationarity of gearbox and bearing vibration signals due to modulation and the suitability of cyclostationary analysis to extract modulation features of such signals, the explicit expressions of cyclic correlation and cyclic spectrum of general AM-FM signals are derived and 


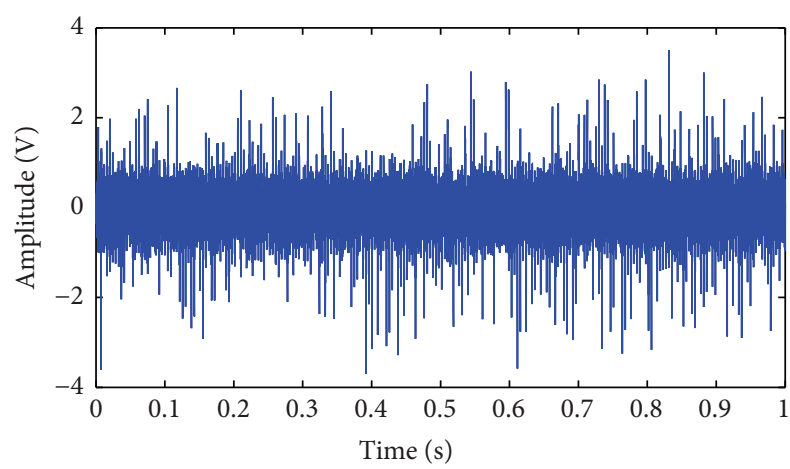

(a) Waveform

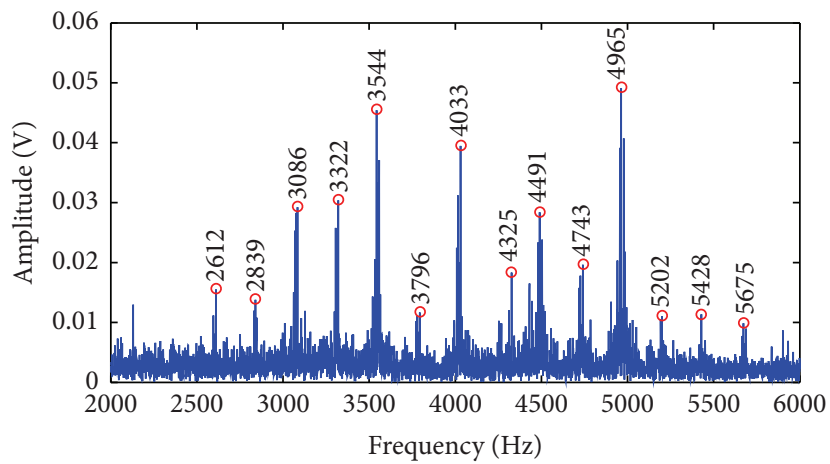

(c) Zoomed-in Fourier spectrum

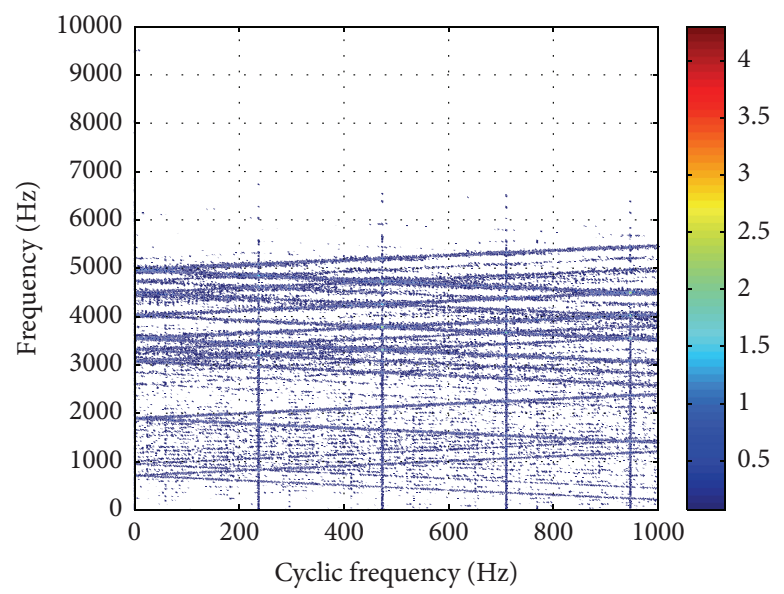

(e) Cyclic spectrum

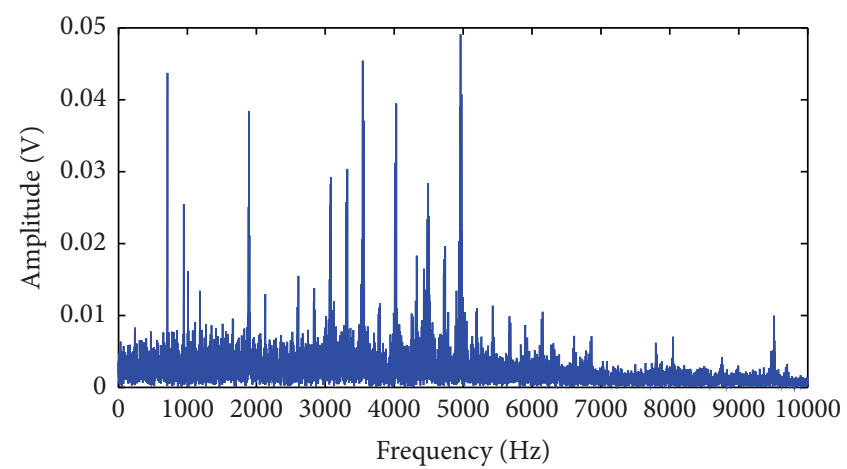

(b) Fourier spectrum

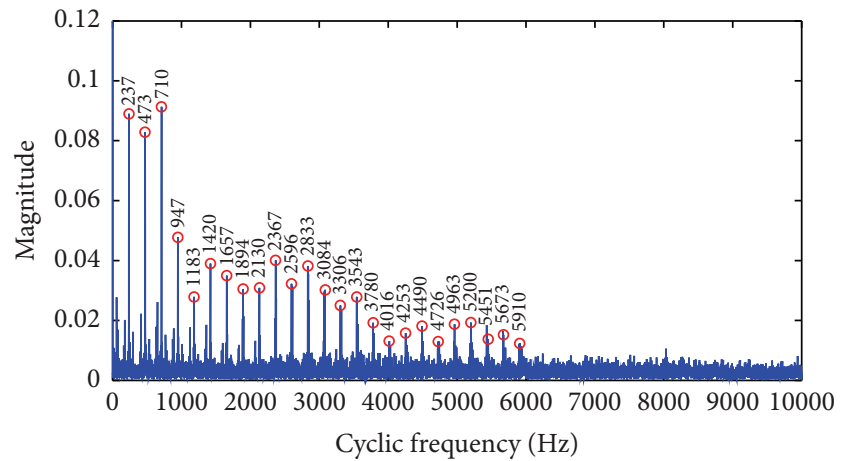

(d) Cyclic correlation 0 lag slice

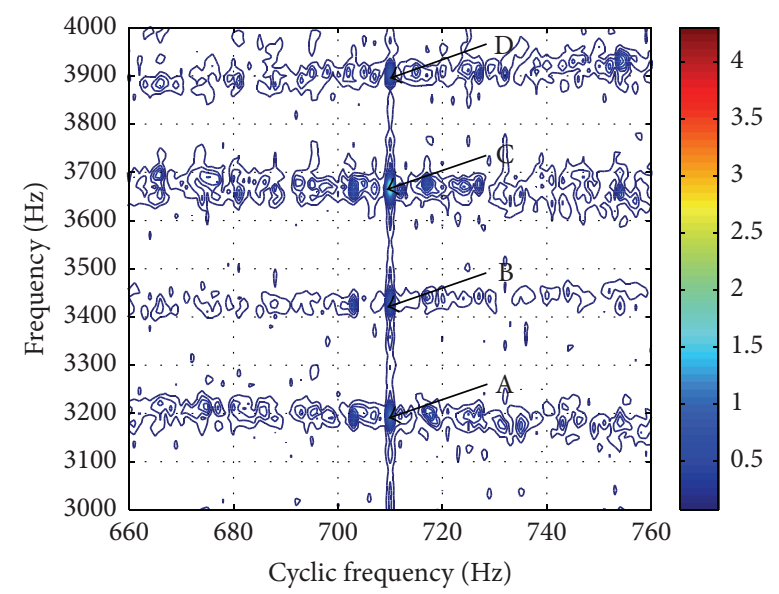

(f) Zoomed-in cyclic spectrum

FIGURE 8: Faulty bearing signal.

validated via gearbox and bearing lab experimental signal analysis. According to the theoretical derivations, the cyclic frequency locations of peaks in cyclic correlation and cyclic spectrum directly correspond to the modulating frequency (i.e., the gear or bearing fault characteristic frequency) and harmonics. Therefore, cyclostationary analysis offers an effective approach to gearbox and bearing fault feature extraction.

\section{Conflict of Interests}

The authors declare that there is no conflict of interests regarding the publication of this paper.

\section{Acknowledgments}

This work is supported by National Natural Science Foundation of China (11272047, 51475038), Program for New Century Excellent Talents in University (NCET-12-0775), and CETIM (Centre des Etudes Techniques des Industries Mécaniques de Senlis), France. The authors would like to thank the reviewers for their valuable comments and suggestions.

\section{References}

[1] R. Shao, W. Hu, and J. Li, "Multi-fault feature extraction and diagnosis of gear transmission system using time-frequency 
analysis and wavelet threshold de-noising based on EMD," Shock and Vibration, vol. 20, no. 4, pp. 763-780, 2013.

[2] S. Luo, J. Cheng, and H. Ao, "Application of LCD-SVD technique and CRO-SVM method to fault diagnosis for roller bearing," Shock and Vibration, vol. 2015, Article ID 847802, 8 pages, 2015.

[3] R. Yan, M. Shan, J. Cui, and Y. Wu, "Mutual informationassisted wavelet function selection for enhanced rolling bearing fault diagnosis," Shock and Vibration, In press.

[4] G. Dalpiaz, A. Rivola, and R. Rubini, "Effectiveness and sensitivity of vibration processing techniques for local fault detection in gears," Mechanical Systems and Signal Processing, vol. 14, no. 3, pp. 387-412, 2000.

[5] C. Capdessus, M. Sidahmed, and J. L. Lacoume, "Cyclostationary processes: application in gear faults early diagnosis," Mechanical Systems and Signal Processing, vol. 14, no. 3, pp. 371$385,2000$.

[6] L. Bouillaut and M. Sidahmed, "Cyclostationary approach and bilinear approach: comparison, applications to early diagnosis for helicopter gearbox and classification method based on hocs," Mechanical Systems and Signal Processing, vol. 15, no. 5, pp. 923943, 2001.

[7] Z. K. Zhu, Z. H. Feng, and F. R. Kong, "Cyclostationarity analysis for gearbox condition monitoring: approaches and effectiveness," Mechanical Systems and Signal Processing, vol. 19, no. 3, pp. 467-482, 2005.

[8] G. Bi, J. Chen, F. C. Zhou, and J. He, "Application of slice spectral correlation density to gear defect detection," Proceedings of the Institution of Mechanical Engineers, Part C: Journal of Mechanical Engineering Science, vol. 220, no. 9, pp. 1385-1392, 2006.

[9] L. Li and L. Qu, "Cyclic statistics in rolling bearing diagnosis," Journal of Sound and Vibration, vol. 267, no. 2, pp. 253-265, 2003.

[10] R. B. Randall, J. Antoni, and S. Chobsaard, "The relationship between spectral correlation and envelope analysis in the diagnostics of bearing faults and other cyclostationary machine signals," Mechanical Systems and Signal Processing, vol. 15, no. 5, pp. 945-962, 2001.

[11] J. Antoni, "Cyclic spectral analysis in practice," Mechanical Systems and Signal Processing, vol. 21, no. 2, pp. 597-630, 2007.

[12] J. Antoni, "Cyclic spectral analysis of rolling-element bearing signals: facts and fictions," Journal of Sound and Vibration, vol. 304, no. 3-5, pp. 497-529, 2007.

[13] A. Raad, J. Antoni, and M. Sidahmed, "Indicators of cyclostationarity: theory and application to gear fault monitoring," Mechanical Systems and Signal Processing, vol. 22, no. 3, pp. 574$587,2008$.

[14] J. Antoni, "Cyclostationarity by examples," Mechanical Systems and Signal Processing, vol. 23, no. 4, pp. 987-1036, 2009.

[15] J. Urbanek, T. Barszcz, and J. Antoni, "Time-frequency approach to extraction of selected second-order cyclostationary vibration components for varying operational conditions," Measurement, vol. 46, no. 4, pp. 1454-1463, 2013.

[16] D. Wang and C. Shen, "An equivalent cyclic energy indicator for bearing performance degradation assessment," Journal of Vibration and Control, 2014.

[17] R. B. Randall, "A new method of modeling gear faults," Journal of Mechanical Design, vol. 104, no. 2, pp. 259-267, 1982.

[18] P. D. McFadden, "Detecting fatigue cracks in gears by amplitude and phase demodulation of the meshing vibration," Journal of
Vibration Acoustics Stress and Reliability in Design, vol. 108, no. 2, pp. $165-170,1986$.

[19] P. D. McFadden and J. D. Smith, "Model for the vibration produced by a single point defect in a rolling element bearing," Journal of Sound and Vibration, vol. 96, no. 1, pp. 69-82, 1984.

[20] P. D. McFadden and J. D. Smith, "The vibration produced by multiple point defects in a rolling element bearing," Journal of Sound and Vibration, vol. 98, no. 2, pp. 263-273, 1985.

[21] M. Liang and I. S. Bozchalooi, "An energy operator approach to joint application of amplitude and frequency-demodulations for bearing fault detection," Mechanical Systems and Signal Processing, vol. 24, no. 5, pp. 1473-1494, 2010.

[22] Z. Feng, M. J. Zuo, R. Hao, and F. Chu, "Gear crack assessment based on cyclic correlation analysis," in Proceedings of the 8th International Conference on Reliability, Maintainability and Safety (ICRMS '09), pp. 1071-1076, Chengdu, China, July 2009.

[23] Z. Feng, R. Hao, F. Chu, M. J. Zuo, and M. El Badaoui, "Application of cyclic spectral analysis to gear damage assessment," in Proceedings of the Prognostics and Health Management Conference, p. 3058, Macau, China, January 2010.

[24] Z. Feng and M. J. Zuo, "Gearbox diagnosis based on cyclic spectral analysis," in Proceedings of the 3rd Annual IEEE Prognostics and System Health Management Conference (PHM '12), pp. 1-5, Beijing, China, May 2012.

[25] W. A. Gardner, Cyclostationarity in Communications and Signal Processing, IEEE Press, New York, NY, USA, 1994.

[26] M. Abramowitz and I. A. Stegun, Handbook of Mathematical Functions With Formulas, Graphs, and Mathematical Tables, Dover Publications, New York, NY, USA, 1972.

[27] H. Qiu, J. Lee, J. Lin, and G. Yu, "Wavelet filter-based weak signature detection method and its application on rolling element bearing prognostics," Journal of Sound and Vibration, vol. 289, no. 4-5, pp. 1066-1090, 2006. 

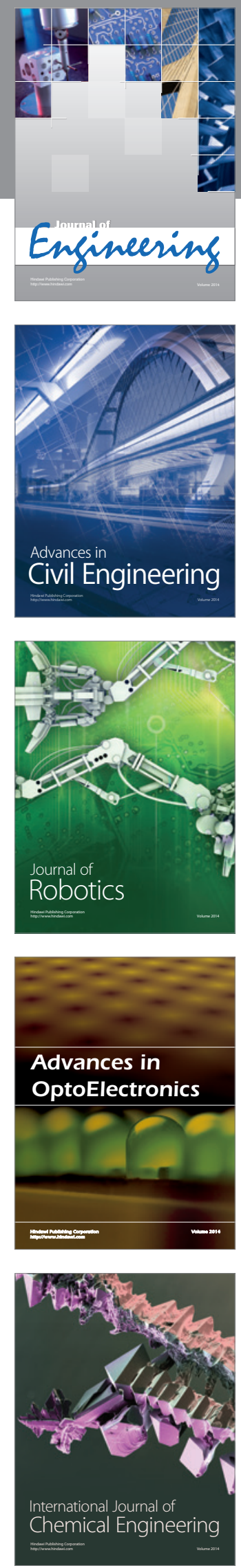

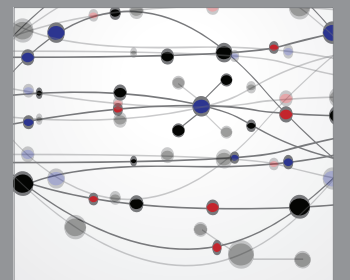

The Scientific World Journal
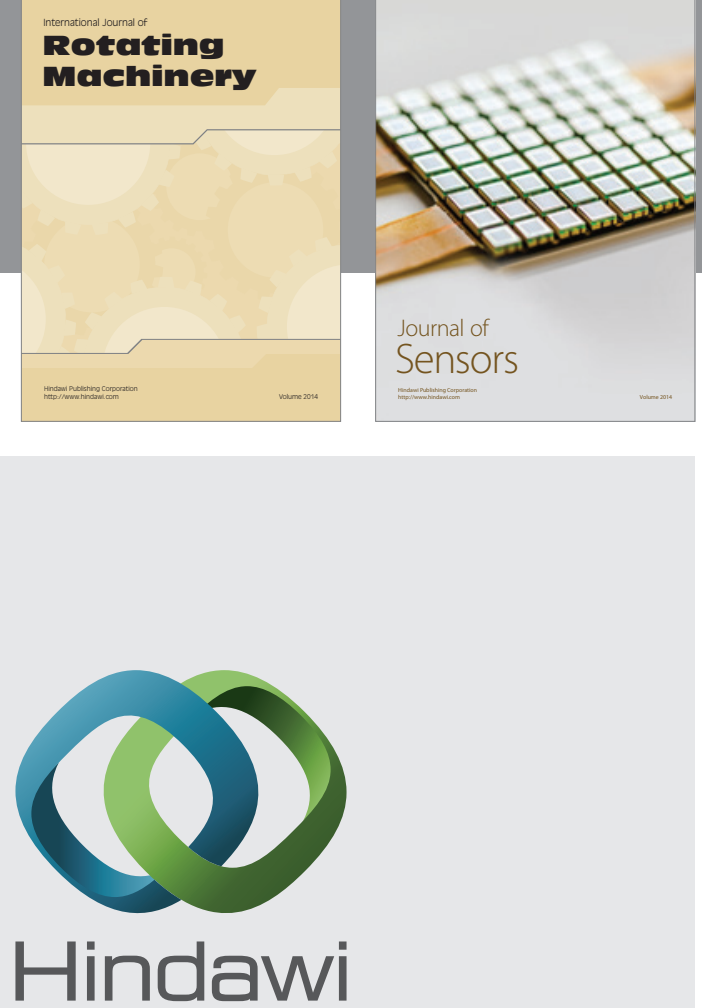

Submit your manuscripts at http://www.hindawi.com
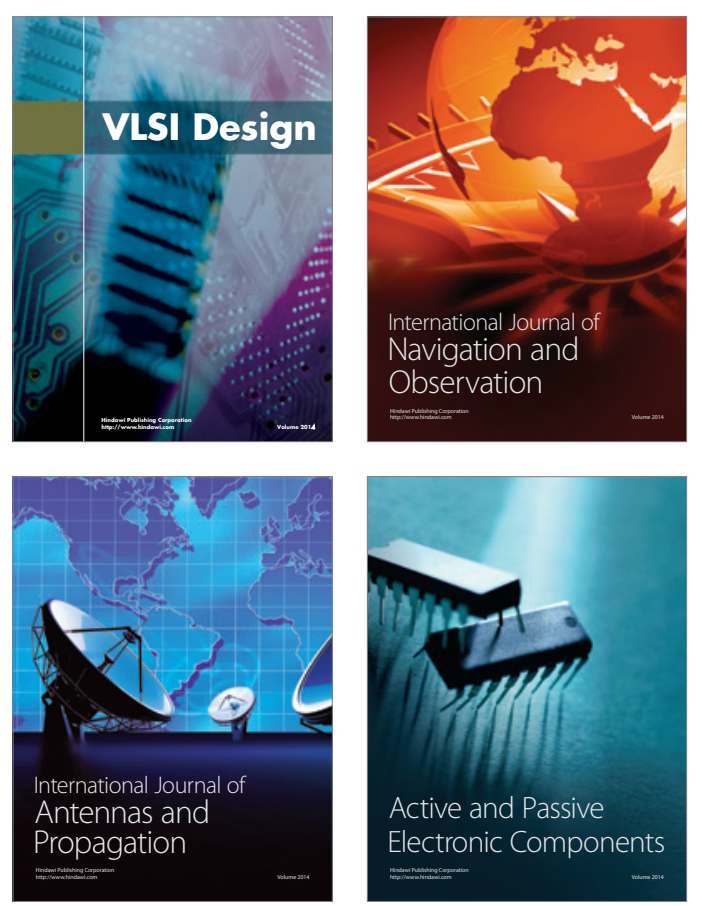
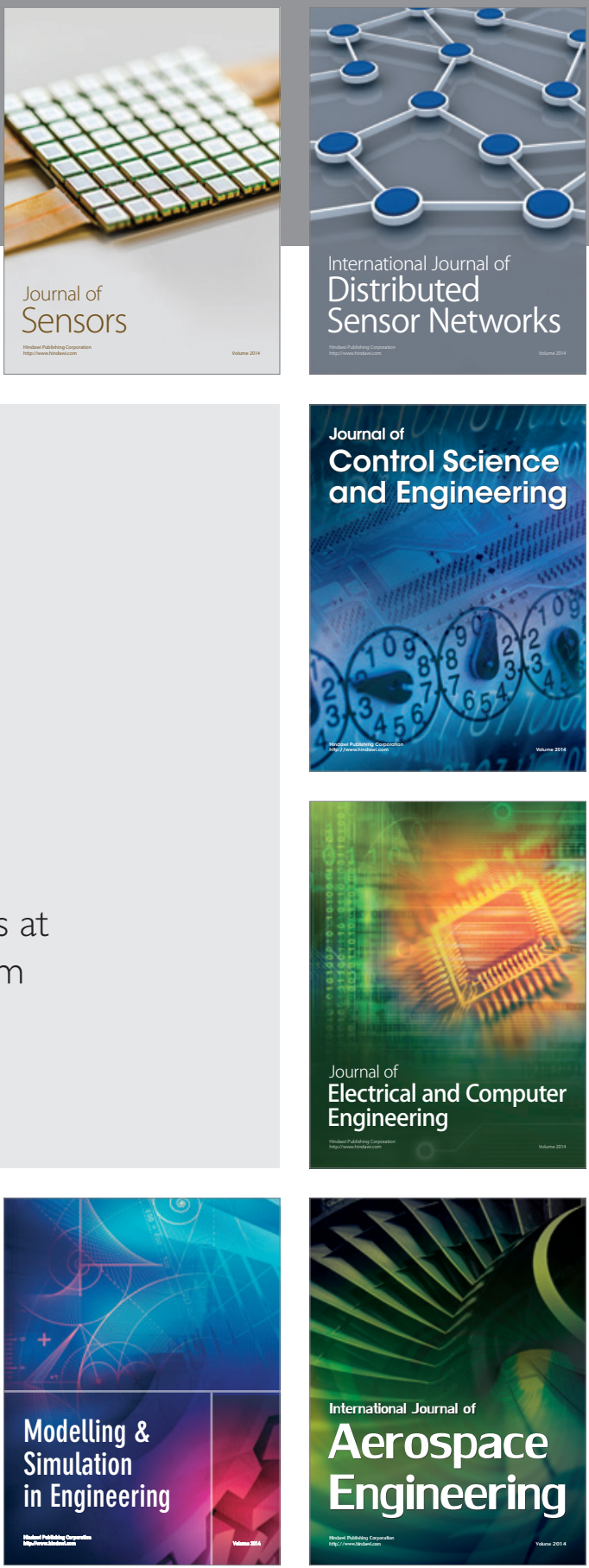

Journal of

Control Science

and Engineering
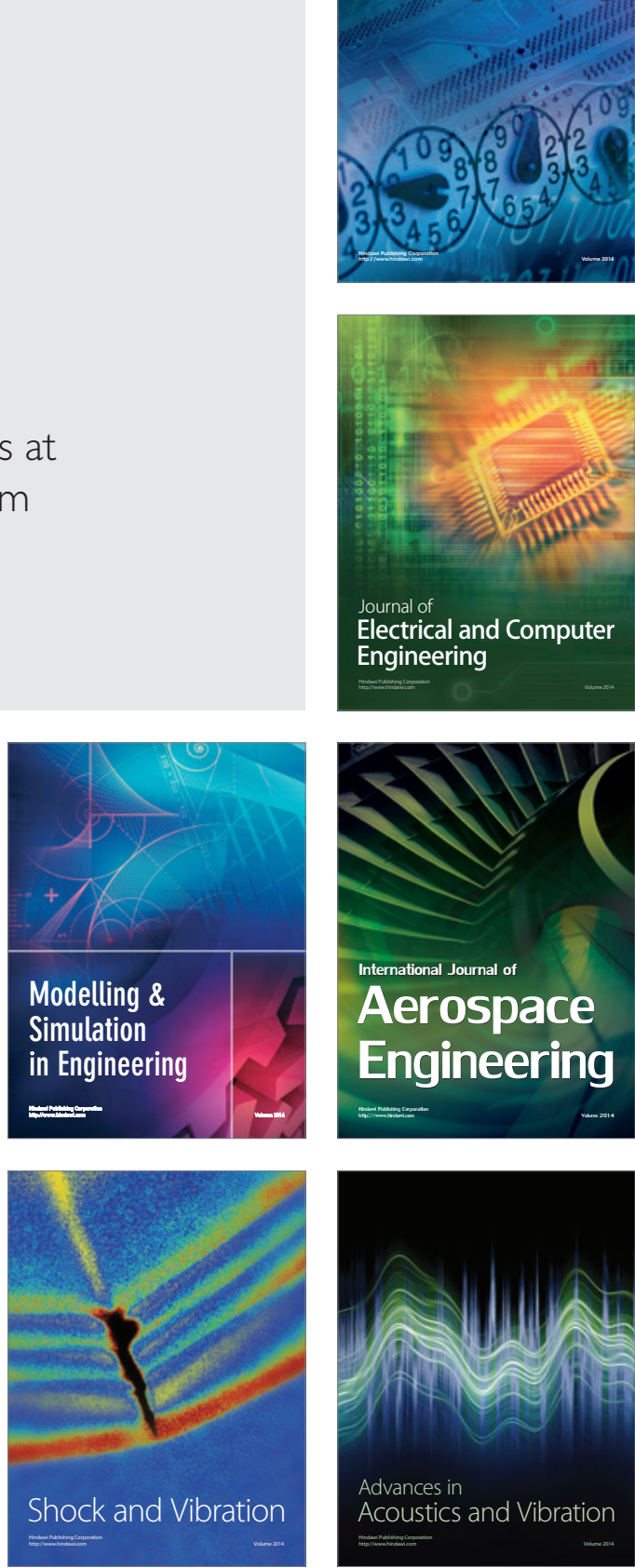